\title{
COMPARATIVE STUDY OF FATTY ACID PROFILES IN PATIN (PANGASIUS MICRONEMUS) AND GABUS (CHANNA STRIATA) FISH OIL AND ITS AUTHENTICATION USING FTIR SPECTROSCOPY COMBINED WITH CHEMOMETRICS
}

\author{
ANGGITA ROSIANA PUTRI ${ }^{1}$, ABDUL ROHMAN ${ }^{1,2 *}$, SUGENG RIYANTO1
}

1Faculty of Pharmacy, Gadjah Mada University, Yogyakarta, 55281, Indonesia, ${ }^{2}$ Research Center of Halal Products, Gadjah Mada University, Yogyakarta, 55281 Indonesia

Email: abdul_kimfar@ugm.ac.id

Received: 15 Jul 2019, Revised and Accepted: 02 Sep 2019

\section{ABSTRACT}

Objective: The aims of this research were to analyse the fatty acids contained in Patin (Pangasius micronemus) and Gabus (Channa striata) fish oils also its authentication using FTIR spectroscopy combined with chemometrics.

Methods: Patin fish oil (PFO) was extracted from patin flesh using the maceration method with petroleum benzene as the solvent, while gabus fish oil (GFO) was purchased from the market in Yogyakarta. The analysis of fatty acid was done using gas chromatography-flame ionization detector (GC-FID). The authentication was performed using FTIR spectrophotometer and chemometrics methods. Principal component analysis (PCA) was used to determine the proximity of oils based on the characteristic similarity. The quantification of adulterated PFO was performed using multivariate calibrations, partial least square (PLS) and principal component regression (PCR). The classification between authentic oils and those adulterated used discriminant analysis (DA).

Results: The level of saturated and polyunsaturated fatty acids in PFO is higher than in GFO. The PLS and PCR methods using the second derivative spectra at wavenumbers of $666-3050 \mathrm{~cm}^{-1}$ offered the highest values of coefficient of determination ( $\left.\mathrm{R}^{2}\right)$ and lowest root means the square error of calibration (RMSEC) and root mean square error of prediction (RMSEP).

Conclusion: The PCA method was successfully used to determine the proximity of oils. Among oils studied, PFO has a similarity fatty acid composition with GFO. The DA method was able to screen pure PFO from adulterated PFO without any misclassification reported. FTIR spectroscopy in combined with chemometrics can be used for authentication and quantification.

Keywords: Fatty acid, Patin fish oil, Gabus fish oil, Chemometrics, Authentication

(c) 2019 The Authors. Published by Innovare Academic Sciences Pvt Ltd. This is an open-access article under the CC BY license (http://creativecommons.org/licenses/by/4.0/) DOI: http://dx.doi.org/10.22159/ijap.2019v11i6.34921

\section{INTRODUCTION}

Fish oils are the major food source of omega-3 fatty acids. Omega-3, mainly consist of eicosapentanoic acid (EPA) and docosahexanoic acid (DHA), have many benefits for human health. Several biological activities of omega-3 have been reported including the prevention of cognitive decline, known as Alzheimer's disease [1], antiinflammatory and anti-oxidant effect [2]. Consuming any food products containing DHA and EPA decreased the risk of coronary heart disease [3] and may help protect against the development of dementia [4].

Patin fish (Pangasius micronemus) and Gabus fish (Channa striata) are freshwater fish that cultivated in Indonesia. Pangasius nasutus flesh was reported to contain DHA and EPA about 1.7-2.8 and 1.0$1.4 \%$ [5]. Other species, Pangasius bocourti were also reported contain DHA and EPA [6] while Gabus fish (Channa striata) oil extracted from fish flesh contain low concentration of DHA and EPA, 0.49 and $1,37 \%$ respectively [7].

Authentication is the determination of the product as right as it is declared [8]. Authentication is needed to protect the consumer from the adulterated product. PFO has higher price than other oils, so it potential to be adulterated with other oils that have lower price. The adulteration practice hard to differentiate physically. So, some analysis methods were developed for authentication purposes. One of the methods that have developed is Fourier transform infrared (FTIR) spectroscopy combined with chemometric.

FTIR Spectroscopy combined with chemometrics could be used for authentication. Every oil contains different fatty acids composition, so it has specific spectra and can be used to differentiate between pure and adulterated oil [9]. The chemometrics techniques commonly used are principal component analysis (PCA), discriminant analysis (DA), and multivariate calibrations. PCA is one of the unsupervised pattern recognition techniques for grouping samples that have a similarity in terms of variables used like fatty acid composition. Partial Least Square (PLS) and principal component regression (PCR) are quantitative analysis methods [10]. Discriminant analysis (DA) is used to find the relationship between the descriptive variable and the qualitative variable [11]. FTIR spectroscopy in combination with chemometrics of PLS, PCR, PCA, and DA, has been reported for the authentication of fish oil of cod liver oil [12, 14]; however, there is no report related to the authentication of Patin fish oil (PFO). The purpose of this research was to analysis fatty acid content from PFO and GFO and to authenticate PFO from GFO using FTIR spectroscopy combined with chemometrics methods.

\section{MATERIALS AND METHODS}

\section{Extraction of patin fish oil (PFO)}

The Patin flesh was separated from other parts of the fish. Then, the flesh was sliced and homogenized using a blender. A $200 \mathrm{~g}$ of samples were maceration with $500 \mathrm{~mL}$ petroleum benzene for $2 \mathrm{~d}$. The extract was filtrated and concentrated using a vacuum rotary evaporator.

\section{Analysis of fatty acid}

Before the analysis of fatty acid using gas chromatography-flame ionization detector (GC-FID), PFO and GFO must be subjected to derivatization into methyl ester form. Derivatization was performed according to the method by Hayati et al. with slight modification [15]. A-0.5 ml PFO and GFO were dissolved with $1 \mathrm{ml} \mathrm{n}$-hexane, and then added with $1 \mathrm{ml} \mathrm{NaOCH}{ }_{3} 0.2 \mathrm{M}$, and the mixture was mixed using a vortex mixer for $1 \mathrm{~min}$. The organic layer $(1 \mu \mathrm{l})$ was injected into GC-FID instrument (GC-2010 Plus, Shimadzu, Japan) using HP88 column ( $100.0 \mathrm{~m}$ length $\mathrm{x} 0.25 \mathrm{~mm}$ inner diameter $\mathrm{x} 0.20 \mu \mathrm{m}$ film thickness, Agilent JandW, USA). Fatty acid was detected using a flame ionization detector (FID). The oven temperature was set at $100{ }^{\circ} \mathrm{C}$ for $5 \mathrm{~min}$ and then ramped until $240^{\circ} \mathrm{C}$ and was held for 15 
min. The injection was performed using split mode with temperature of $260^{\circ} \mathrm{C}$ using helium as carrier gas with flow rate $1.72 \mathrm{ml} / \mathrm{min}$. The percentage of fatty acid was calculated and corrected using correction response factors obtained by analysis of the standard mixture of fatty acid methyl esters (FAME). Quantitative analysis of FA was performed using internal normalization technique.

$$
\text { Percentage (\%) fatty acid } x=\frac{\text { Peak area of fatty acid } x}{\text { Total peak area of all fatty acids }} \times 100 \%
$$

\section{PCA analysis}

For PCA analysis, some vegetable oils (grapeseed oil, red fruit oil, sunflower oil, black seed oil, corn oil, soybean oil, palm oil, candlenut oil, olive oil, and virgin coconut oil), fish oils (gabus fish oil, shark fish oil, codfish oil, salmon fish oil, and Patin fish oil), and animal oils (soft-shelled turtle oil and leech oil) were collected and scanned using FTIR spectrophotometer. As variables, the absorbance values at selected wavenumbers region were used.

\section{Quantification of PFO adulterated with GFO}

To quantification of PFO adulterated with GFO, prepared calibration and validation samples. For the calibration sample, a series of the mixture of PFO and GBO with a concentration range of $0-50 \%(\mathrm{v} / \mathrm{v})$ was made. Then, a set of validation samples covering PFO and GFO samples was also prepared. All calibration and validation samples were subjected to FTIR spectrophotometer.

\section{Discriminant analysis}

For discriminant analysis (DA), a training set of pure PFO and adulterated PFO was prepared and subjected to FTIR spectra measurement. The $100 \%$ PFO was assigned as "pure" PFO and PFO adulterated with GFO was assigned with "adulterated".

\section{FTIR spectra analysis}

The Analysis of fatty acid using FTIR (Thermo Scientific Nicolet iS10, Madison, WI) instrument with Omnic software. The measurement was performed in the middle infrared region of $4000-650 \mathrm{~cm}^{-1}$ with 32 scans at the resolution of $16 \mathrm{~cm}^{-1}$. The background scan was performed to do to reduce the effect of the reference spectrum of the air. Before and after analyzing the sample, ATR crystal was cleaned with acetone p. a. Triplicate scanning of samples was performed.

\section{Chemometrics analysis}

The chemometrics of PCA for the classification among oils was done using the Minitab software version 18. The data analysis involving multivariate calibrations of PLS and PCR as well as DA were performed using TQ Analyst software version 9 (Thermo Fisher Scientific Inc.).

\section{RESULTS AND DISCUSSION}

\section{Fatty acid composition}

Fatty acids composed of fats and oils consisted of saturated fatty acid (SFA), monounsaturated fatty acid (MUFA), and polyunsaturated fatty acid (PUFA). SFA is fatty acid in which all carbon chains are single bond, MUFA contains one double bond, and PUFA is fatty acid in which carbon chain contains more than one double bond. SFA contained in PFO was higher than corresponding MUFA and PUFA. In addition, SFA and PUFA contents in PFO was higher than those in GFO.

The main SFA in PFO and GFO was hexadecanoic acid (table 1). While the highest content MUFA in PFO and GFO was found in oleic acid 31 and $40 \%$. Linoleic acid was the major PUFA content in PFO and GFO, 14 and $9 \%$ respectively. The difference of fatty acid content in PFO and GFO could be influenced by the feed as patin fish feed contain high SFA and PUFA content [6]. Docosahexanoic acid (DHA) and eicosapentaenoic acid (EPA) include in PUFA. The difference of fatty acid content among PFO and GFO could be used to analysis PFO in adulterate with GFO.

\section{Principal component analysis}

PCA is a multivariate statistical method with unsupervised nonparametric. PCA method using data from measurement to make relationship between some samples based on similarities content. PCA method was reduced many variables in data and make a new set variable known as Principal Components. PCA usually used two principal components (PCs) to make a score plot. Score plot exhibits the space between samples. Samples that have similar content will place in close space [16].

Table 1: Fatty acid composition from PFO and GFO

\begin{tabular}{|c|c|c|}
\hline Fatty acid & PFO & GFO \\
\hline Butanoic acid (C4:0) & 1.792 & 0.508 \\
\hline Hexanoic acid (C6:0) & 0.000 & 0.561 \\
\hline Decanoic acid (C10:0) & 0.000 & 0.208 \\
\hline Undecanoic acid (C11:0) & 0.199 & 0.000 \\
\hline Dodecanoic acid (C12:0) & 0.263 & 0.229 \\
\hline Tridecanoic acid (C13:0) & 0.133 & 0.000 \\
\hline Tetradecanoic acid (C14:0) & 2.920 & 0.869 \\
\hline Pentadecanoic acid (C15:0) & 0.135 & 0.000 \\
\hline Hexadecanoic acid (C16:0) & 22.814 & 26.830 \\
\hline Heptadecanoic acid (C17:0) & 0.741 & 0.284 \\
\hline Octadecanoic acid (C18:0) & 5.464 & 3.106 \\
\hline Eicosanoic acid (C20:0) & 1.070 & 0.524 \\
\hline Heneicosanoic acid (C21:0) & 1.491 & 1.012 \\
\hline Docosanoic acid (C22:0) & 1.477 & 0.714 \\
\hline Tricosanoic acid (C23:0) & 0.440 & 0.454 \\
\hline Tetracosanoic acid (C24:0) & 0.825 & 0.254 \\
\hline Total of saturated fatty acid & 39.764 & 35.553 \\
\hline Palmitoleic acid (C16:1) & 1.272 & 0.608 \\
\hline Oleic acid (C18:1) & 31.078 & 40.834 \\
\hline Eicosenoic acid (C20:1) & 1.408 & 0.599 \\
\hline Erucic acid (C22:1) & 0.911 & 0.476 \\
\hline Nervonic acid (C24:1) & 3.011 & 1.488 \\
\hline Total of monounsaturated fatty acid & 37.680 & 44.005 \\
\hline Linoleic acid (C18:2) & 14.703 & 9.901 \\
\hline Eicosadienoic acid (C20:2) & 1.842 & 0.799 \\
\hline Docosadienoic acid (C22:2) & 0.941 & 1.424 \\
\hline Gamma-Linoleic acid (C18:3) & 0.491 & 0.850 \\
\hline Eicosatrienoic acid (C20:3) & 0.557 & 0.130 \\
\hline Eicosatetranoic acid (C20:4) & 1.283 & 0.312 \\
\hline Eicosapentanoic acid (C20:5) & 0.447 & 4.812 \\
\hline Docosahexanoic acid (C22:6) & 2.076 & 2.036 \\
\hline Total of polyunsaturated fatty acid & 22.340 & 20.264 \\
\hline
\end{tabular}


Some samples consist of edible oils, fish oils, and animal oil was scanned using FTIR spectrophotometer. Then the spectra were analysed using PCA method. PCA method was done using Minitab version 18. The result was showed in fig. 1 . According to fig. 1, the PFO was close to GFO that is implied the PFO has similar fatty acid content with GFO as supported by the dendrogram in fig. 2 .

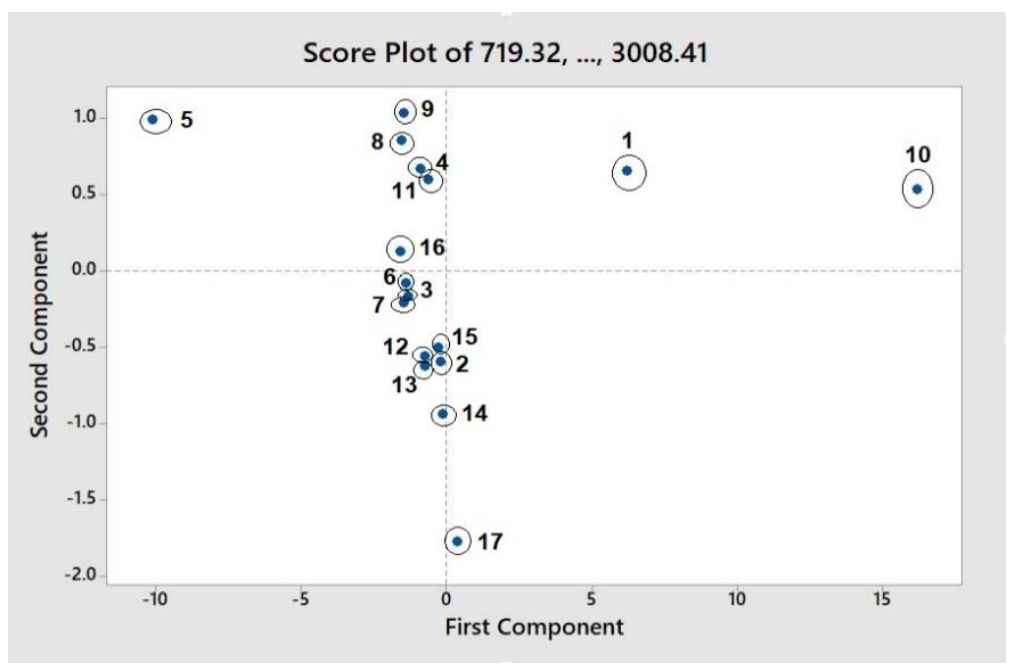

Fig. 1: The score plot of some oils. 1: grapeseed oil, 2: red fruit oil, 3: soft-shelled turtle oil, 4: sunflower oil, 5: habbatussauda oil, 6: gabus fish oil, 7: shark fish oil, 8: cod fish oil, 9: salmon fish oil, 10: corn oil, 11: soybean oil, 12: palm oil, 13: candlenut oil, 14: leech oil, 15: olive oil, 16: patin fish oil, 17: virgin coconut oil

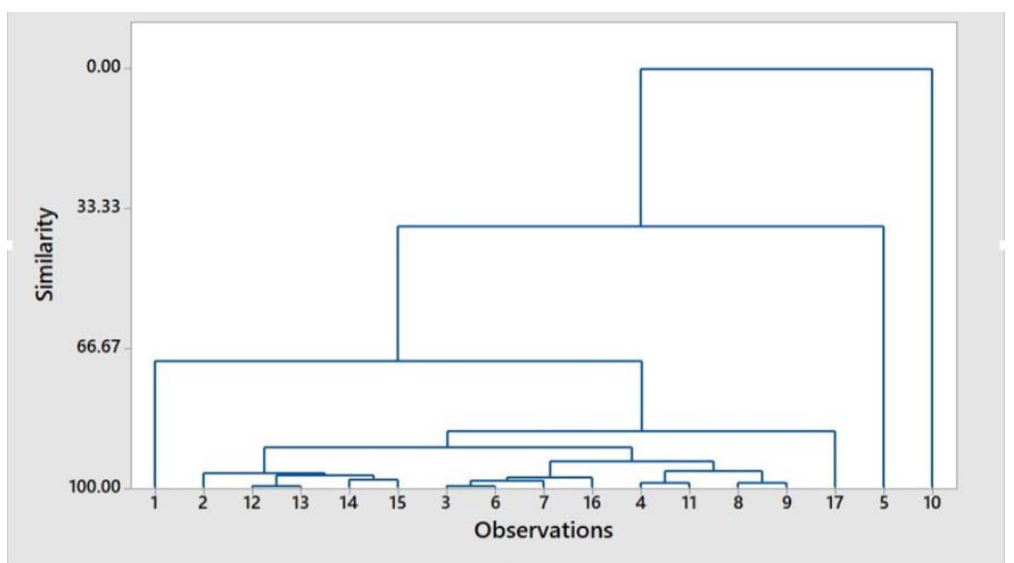

Fig. 2: Dendrogram of some edible oils, fish oils, and animal oils. 1: grapeseed oil, 2: red fruit oil, 3: soft-shelled turtle oil, 4: sunflower oil, 5: habbatussauda oil, 6: gabus fish oil, 7: shark fish oil, 8: cod fish oil, 9: salmon fish oil, 10: corn oil, 11: soybean oil, 12: palm oil, 13: candlenut oil, 14: leech oil, 15: olive oil, 16: patin fish oil, 17: virgin coconut oil

Table 2: Vibration of functional groups in PFO and GFO at certain wavenumber [17]

\begin{tabular}{ll}
\hline Wavenumber $\left(\mathbf{c m}^{-1}\right)$ & Functional group \\
\hline 3005 & cis $\mathrm{C}=\mathrm{C}$ stretching \\
2954 & $-\mathrm{CH}_{3}$ asymmetrical \\
2922 and 2854 & Asymmetric and symmetric methylene $\left(-\mathrm{CH}_{2}\right)$ group \\
1744 & $-\mathrm{C}=\mathrm{O}$ stretch \\
1460 & $-\mathrm{CH}_{2}$ and- $\mathrm{CH}_{3}$ bending vibration \\
1374 & $-\mathrm{CH} 2$ group bending vibration \\
1233 and 1157 & $-\mathrm{C}-\mathrm{O}$ stretching \\
1113 & Stretching vibration of $-\mathrm{C}-\mathrm{O}$ ester \\
960 & Bending vibration of $-\mathrm{CH}$ \\
721 & Rocking vibration of methylene $\left(-\mathrm{CH}_{2}\right)$ \\
\hline
\end{tabular}

\section{Spectra analysis}

Table 2 shows the vibration of a functional group on fatty acid contained in PFO and GFO. Every functional group was signified at certain wavenumber. The FTIR spectra of PFO and GFO were shown in fig. 3. There is a difference between PFO and GFO. PFO has a peak at $2954 \mathrm{~cm}^{-1}$ and GFO have no peak, so the area could be used to detection adulteration of PFO.

\section{Quantification}

To quantification PFO that adulterated with GFO using partial least square (PLS) and principle component regression (PCR). PLS and PCR was done using TQ analyst. To quantification, 27 samples contain PFO mixed GFO was prepared with range concentration $0-50 \%$. The spectral regions that used to quantification are at $666-3050 \mathrm{~cm}^{-1}$. The regions were chosen by 
optimization process to get the highest values of $R^{2}$ for calibration and validation [13]

The PLS and PCR method were the factor analysis method. In these methods were built a mathematical model using calibration, concentration, and analytical signal from the samples. From measurement the concentration of samples using FTIR, then would get the actual concentration sample. PLS method using spectral and concentration data then make information in the new space of principal component. While PCR method using all information from spectral data and concentration sample into a model in one step [18]

The overlay FTIR spectra of PFO and GFO shown in fig. 4. In PLS and PCR method using normal, first derivative and second derivative spectra to get the best model for quantification and calibration. These derivative technique could differentiate the spectra from mixed samples by increasing the resolution. From PLS and PCR methods would obtain $\mathrm{R}^{2}$ value, RMSEC, and RMSEP. Root mean square of calibration (RMSEC) was used to evaluate error in calibration model and the root mean square of prediction (RMSEP) was used to evaluate the validation model. PLS and PCR method using "leave one out" validation technique by remove one of the standard samples [13].

Second derivative spectra give the best model calibration in both PLS and PCR model with the highest $\mathrm{R}^{2}$, lowest RMSEC and RMSEP (table 3). In the PLS and PCR method, the $\mathrm{R}^{2}$ value of calibration were 0,9975 and 0,9949 . The highest of $R^{2}$ value and the low of RMSEC and RMSEP indicated that calibration and validation models using PLS and PCR method with second derivative spectra were accurate and precision to measure the concentration of PFO that adulterated with GFO.

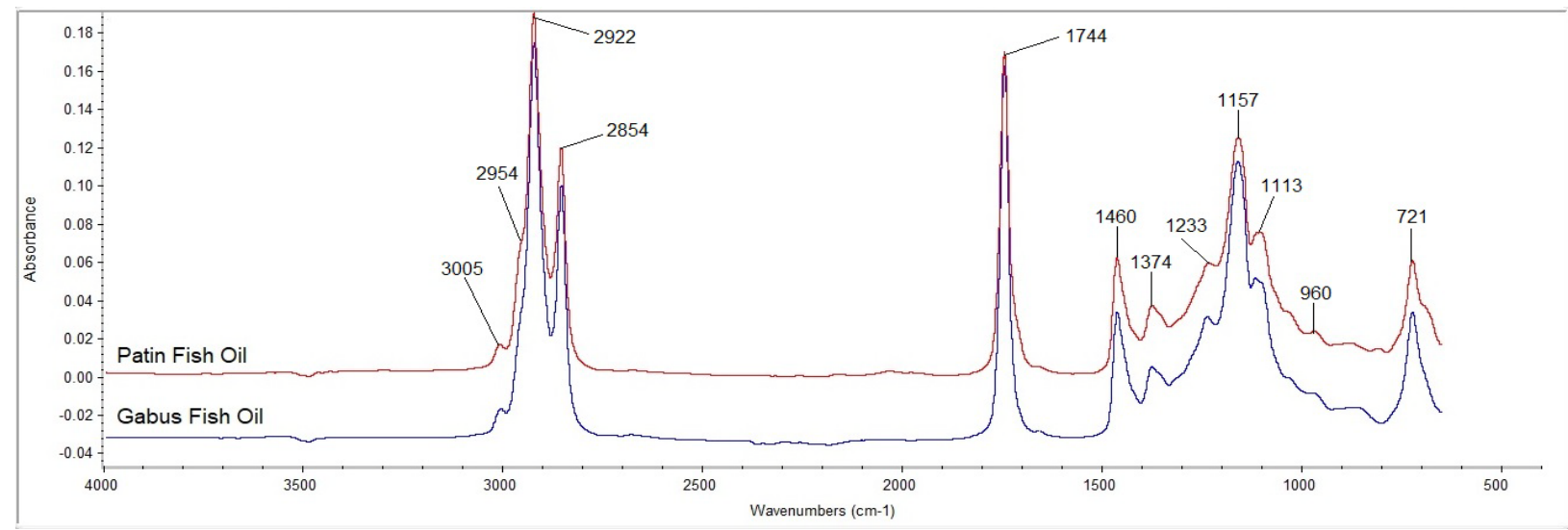

Fig. 3: FTIR spectra of patin fish oil (PFO) and gabus fish oil (GFO) at wavenumber of 4000-650 $\mathrm{cm}^{-1}$

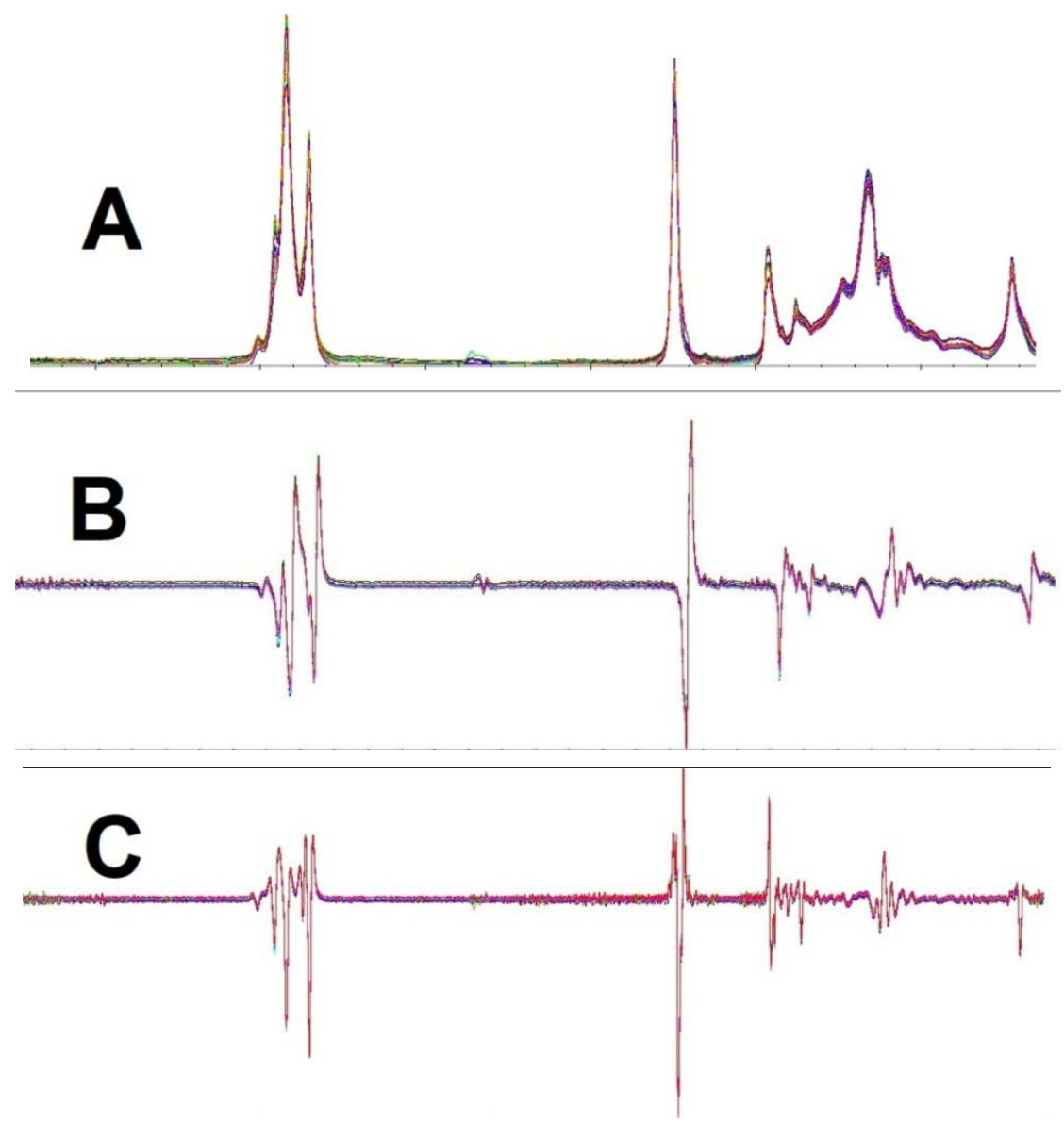

Fig. 4: Overlay the FTIR spectra of PFO and GFO. (A) Normal spectra (B) first derivative spectra (C) second derivative spectra 
Table 3: PLS and PCR performance for analysis the mixing component of PFO and GFO

\begin{tabular}{|c|c|c|c|c|c|c|c|}
\hline \multirow[t]{2}{*}{ Model } & \multirow[t]{2}{*}{ Spectra } & \multicolumn{2}{|l|}{ Equation } & \multicolumn{2}{|l|}{$\mathbf{R}^{2}$} & \multirow[t]{2}{*}{ RMSEC } & \multirow[t]{2}{*}{ RMSEP } \\
\hline & & Calibration & Validation & Calibration & Validation & & \\
\hline \multirow{3}{*}{ PLS } & Normal & $y=0.9978 x+0.1728$ & $y=2.376 x-135.26$ & 0.9978 & 0.9806 & 0.683 & 1.720 \\
\hline & 1st derivative & $y=0.9958 x+0.3308$ & $y=1.031 x-3.6315$ & 0.9958 & 0.9906 & 0.944 & 1.730 \\
\hline & 2nd derivative & $y=0.9995 x+0.0412$ & $y=1.0354 x-3.9392$ & 0.9995 & 0.9929 & 0.339 & 1.570 \\
\hline \multirow{3}{*}{ PCR } & Normal & $y=0.9951 x+0.3886$ & $y=1.016 x-1.3328$ & 0.9951 & 0.9916 & 1.030 & 1.900 \\
\hline & 1st derivative & $y=0.9947 x+0.4172$ & $y=1.013 x-1.1824$ & 0.9947 & 0.9916 & 1.070 & 1.860 \\
\hline & 2nd derivative & $y=0.9936 x+0.478$ & $y=1.006 x-0.7339$ & 0.9958 & 0.9935 & 0.987 & 1.760 \\
\hline
\end{tabular}

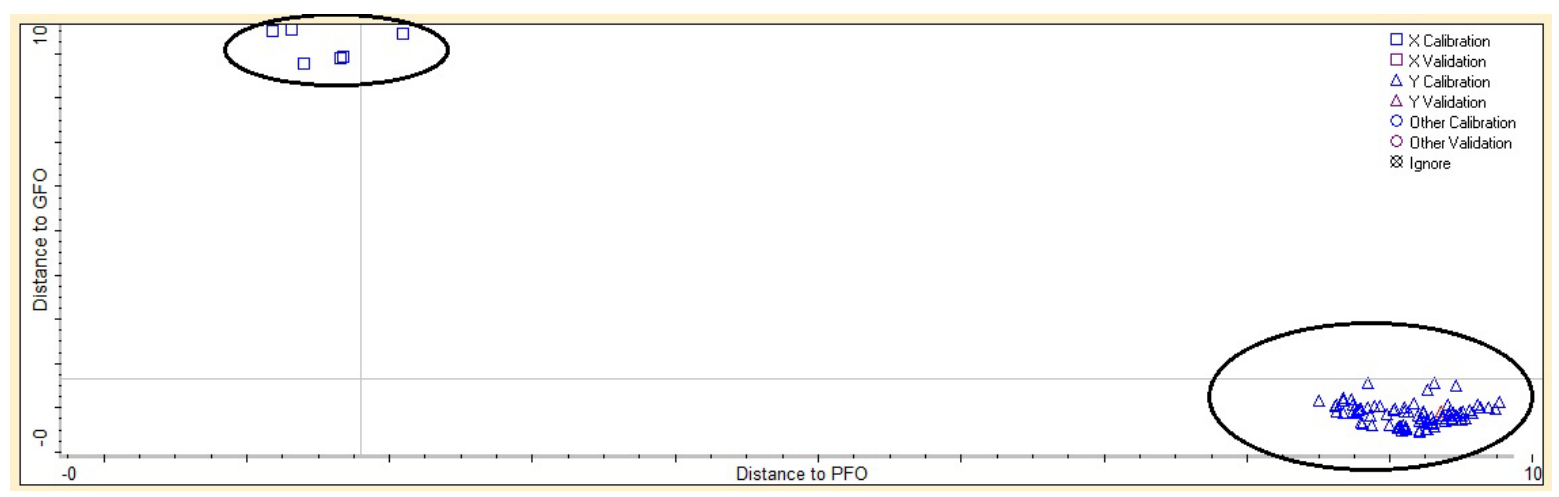

Fig. 5: The Coomans plot of Patin Fish Oil (PFO) and gabus fish oil (GFO): ( $\Delta$ ) PFO adulterated with GFO ( $\square$ ) pure PFO

\section{Discriminant analysis}

Discriminant analysis was a method used to determine among the samples contain pure PFO and adulterated PFO by computing the distance from each group center using Mahalanobis distance units. From this method could be used to predicted the unknown samples whether the samples were pure or adulterate [11]. Discriminant analysis using wavenumber at regions $666-3050 \mathrm{~cm}^{-1}$. The process of discriminant analysis consists of, grouping the PFO spectra and PFO adulterated with GFO spectra be two groups. Then these groups were classified using DA technique and Coomans plot. The Coomans plot is the method to show the group of pure PFO and adulterated PFO. This result is from calculating two principle component (PC) models and then plotting the residual distances of samples from each two models [19].

Fig. 5 exhibits the Coomans Plot for PFO and PFO adulterated with GFO and show the group of pure PFO and group of adulterated PFO. The $\mathrm{x}$-axis show the Mahalanobis distance for PFO and the $\mathrm{y}$-axis show the distance of PFO that adulterated with GFO. The Mahalanobis distance is the best technique to a grouping of sample that have similarity content. These DA models can grouping with $100 \%$ accurate for the samples. So, this method is the best method for grouping the pure samples and adulterate samples.

\section{CONCLUSION}

PFO and GFO were contain different fatty acid. PFO have SFA and PUFA content more than GFO. The difference of fatty acid content could be used to analysis pure PFO and adulterated PFO. FTIR spectroscopy that combined with chemometrics were the best method for authentication. Using PCA method would get the group of samples that have similarity of fatty acid content. PLS and PCR method using second derivative spectra give the highest value of $\mathrm{R}^{2}$ and lowest RMSEC and RMSEP. DA was the the best technique to grouping pure PFO and adulterated PFO accurately.

\section{ACKNOWLEDGMENT}

Author thanks to The Ministry of Research, Technology, and Higher Education of the Republic of Indonesia for its scholarship so this research can be done.

\section{AUTHORS CONTRIBUTIONS}

All the authors have contributed equally

\section{CONFLICT OF INTERESTS}

Declared none

\section{REFERENCES}

1. Hooper, Claudie P, De Souto Barreto M, Pahor M, Weiner B, Vellas. The relationship of omega 3 polyunsaturated fatty acids in red blood cell membranes with cognitive function and brain structure: a review focussed on Alzheimer's disease. J Prev Alzheimer's 2018;5:78-84.

2. Song C, Shieh CH, Wu YS, Kalueff A, Gaikwad S, Su KP. The role of omega-3 polyunsaturated fatty acids eicosapentaenoic and docosahexaenoic acids in the treatment of major depression and Alzheimer's disease: acting separately or synergistically? Prog Lipid Res 2016;62:41-54.

3. Stark KD, Van Elswyk ME, Higgins MR, Weatherford CA, Salem Jr N. Global survey of the omega-3 fatty acids, docosahexaenoic acid and eicosapentaenoic acid in the blood stream of healthy adults. Prog Lipid Res 2016;63:132-52.

4. Ammann EM, Pottala JV, Robinson JG, Espeland MA, Harris WS. Erythrocyte omega-3 fatty acids are inversely associated with incident dementia: secondary analyses of longitudinal data from the Women's Health Initiative Memory Study (WHIMS)," prostaglandins leukot. Essent Fatty Acids 2017;121:68-75.

5. Hashim RB, Jamil EF, Zulkipli FH, Daud JM. Fatty acid compositions of silver catfish, pangasius sp. farmed in several rivers of pahang, Malaysia. J Oleo Sci 2015;64:205-9.

6. Thammapat P, Raviyan P, Siriamornpun S. Proximate and fatty acids composition of the muscles and viscera of Asian catfish (Pangasius bocourti). Food Chem 2010;122:223-7.

7. Molla MR, Asaduzzaman AK, Mia MA, Uddin M, Biswas S, Uddin MS. Nutritional status, characterization and fatty acid composition of oil and lecithin isolated from freshwater fish shoul (Channa striata). Int Nutr Food Sci 2016;5:9.

8. Rodionova OY, Titova AV, Pomerantsev AL. Discriminant analysis is an inappropriate method of authentication. TrAC Trends Anal Chem 2016;78:17-22.

9. Rohman A, Che Man YBC. Fourier transform infrared (FTIR) spectroscopy for analysis of extra virgin olive oil adulterated with palm oil. Food Res Int 2010;43:886-92.

10. Paradkar MM, Irudayaraj JA. Rapid FTIR spectroscopic method for estimation of caffeine in soft drinks and total methylxanthines in tea and coffee. J Food Sci 2002;67:2507-11. 
11. Ballabio D, Todeschini R. Multivariate classification for qualitative analysis, in: In. DW Sun. (Ed). Infrared spectroscopy for food quality analysis and control. Elsevier, London; 2009. p. 83-104.

12. Rohman A, Che Man YB. Analysis of cod-liver oil adulteration using fourier transform infrared (FTIR) spectroscopy. J Am Oil Chem Soc 2009;86:1149-53.

13. Rohman A, Che Man YB. Application of fourier transform infrared (FT-IR) spectroscopy combined with chemometrics for authentication of cod-liver oil. Vib Spectrosc 2011; 55:141-5.

14. Rohman A, Widyaningtyas R, Amalia F. Authentication of cod liver oil from selected edible oils using FTIR spectrophotometry and chemometrics. Int Food Res J 2017;4:1362-7.

15. Nor Hayati I, Che Man YB, Tan CP, Nor Aini. Physicochemical characteristics of soybean oil, palm kernel olein, and their binary blends. Int J Food Sci Technol 2009;44:152-61.
16. Wang K, Yuan Y, Han S, Yang H. Application of attenuated total reflectance fourier transform infrared (ATR-FTIR) and principal component analysis (PCA) for quick identifying of the bitumen produced by different manufacturers. Road Mater Pavement Desvol 2018;19:1940-9.

17. Guillen MD, Cabo N. Characterization of edible oils and lard by fourier transform infrared spectroscopy. Relationships between composition and frequency of concrete bands in the fingerprint region. J Am Oil Chem Soc 1997;74:1281-6.

18. De Luca M, Ioele G, Spatari C, Ragno G. Optimization of wavelength range and data interval in the chemometric analysis of complex pharmaceutical mixtures. J Pharm Anal 2016;6:64-9.

19. Gurdeniz G, Tokatli F, Ozen B. Differentiation of mixtures of monovarietal olive oils by mid-infrared spectroscopy and chemometrics. Eur J Lipid Sci Technol 2007;109: 1194-202. 